\title{
Design Optimization of Linear Generator for a Hydrokinetic Energy Converter
}

\author{
Krishna Manasa Rao ${ }^{1}$, Ashray Gururaja Manur ${ }^{2}$ \\ ${ }^{1}$ Department of Electrical and Electronics Engineering, Sri Jayachamarajendra College of Engineering, India \\ ${ }^{2}$ Department of Electrical and Electronics Engineering, Sri Jayachamarajendra College of Engineering, India
}

\begin{abstract}
The power take off (PTO) mechanism for a vortex induced vibration (VIV) based hydrokinetic energy converter discussed here is a linear generator. The power take off system is one of the most important systems of the converter which is responsible for the conversion of mechanical energy into useful electrical energy. This paper deals with optimization of the design of the linear generator in areas such as materials of construction, engineering design, water proofing, magnetic configuration, air-gap reduction, durability and cost-effectiveness. The linear generator consists of moving and non-moving parts and its working environment is a fluid which is generally water. The main challenges include complete water-proofing of the entire linear generator, reduction of weight of the moving part, smoother motion of the moving part over the non-moving part, corrosion protection of all metal parts and sustenance of intensity of the magnetic field strength. The proposed design aims to address these challenges and increase the efficiency of the overall system.
\end{abstract}

Keywords - Halbach Array, Hydrokinetic Energy, Linear Generator, Power Take off System, Vortex Induced Vibrations

\section{INTRODUCTION}

Hydrokinetic energy converter discussed here uses the concept of vortex induced vibrations. Extensive studies have been carried out to better understand fluid-structure interaction in case of vortex induced vibrations [1-3]. It is desirable to reach the phenomenon of lock in [4] where the vortex shedding frequency is in the near range of natural frequency. A bluff body, usually a cylinder, is introduced in a free stream which causes boundary layer separation. This results in unequal pressure distribution around the bluff body eventually leading to formation of vortices. The vortex shedding pattern is not symmetrical because of which there is a net force which results in the up and down motion of the bluff body. To convert this up and down motion of the cylinder to useful electrical energy is the role of the power take off mechanism which is a linear generator in this case. The linear generator basically works on the principle of electromagnetic induction. The main parts of the linear generator are the core-coil and magnet assembly. Both these assemblies will be in water with core-coil assembly fixed and the magnet assembly in motion.The factors affecting the overall design of the linear generator are as follows

- Strength and configuration of magnets used

- Air gap between core-coil assembly and the magnet housing

- Number of turns of wire used in windings

- Smoothness of motion of the magnet housing and bluff body

- Weight of the magnet housing and bluff body

- Water proofing of the magnet housing and core-coil assembly

- Anti-corrosion protection of iron core

\section{MAgnet ARRAY Housing}

Strong magnets are vital to introduce a magnetic field of high intensity. The magnetic field should be concentrated at the center of the magnet housing where the core-coil assembly is setup. In this experiment cuboidal neodymium magnets of grade N45 were used. The magnets were arranged in Halbach magnet array configuration. The dimensions of the magnets used were $1^{\prime \prime} \times 1^{\prime \prime} \times 0.5^{\prime \prime}$. They are magnetized through the thickness with the poles on the $1^{\text {" }} \times 1^{\prime \prime}$ surfaces. The material of construction proposed for construction of magnetic array housing to hold the magnets in Halbach array configuration is High Density Poly Ethylene (HDPE). It is essential to use a material whose permeability matches closely with air in order to ensure minimum interference with the magnetic field of the Halbach magnet array configuration.Eight neodymium magnets were used which were arranged as shown in Fig 1[5] which results in highly uniform field inside the array and near cancellation of the field outside the array. 


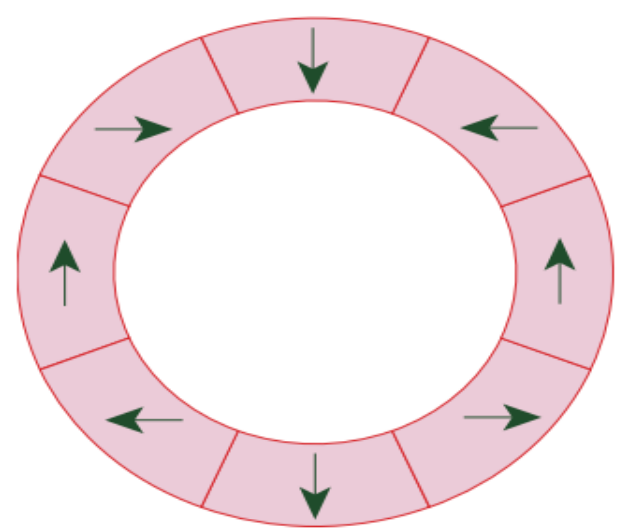

Figure1: Halbach array configuration for 8 magnet system

The dipole field inside the array [5] is given by:

$B_{o}=B_{r} C_{N} \ln \frac{r_{2}}{r_{1}}$

Where

$\mathrm{B}_{\mathrm{r}}=$ residual magnetic field

$\mathrm{r}_{2}=$ outer radius of array

$\mathrm{r}_{1}=$ inner radius of array

$\mathrm{M}=$ number of magnets

$C_{N}=\frac{\sin \frac{2 \pi}{M}}{\frac{2 \pi}{M}}$

For our system, $\mathrm{M}=8$ and hence we get $\mathrm{C}_{\mathrm{N}}=0.9$.

Table 1: Magnet Specifications

\begin{tabular}{|l|l|}
\hline Magnet Specification & Specification Value \\
\hline Grade & $\mathrm{N} 45$ \\
\hline Dimension & $1^{\prime \prime} \times 1^{\prime \prime} \times 0.5^{\prime \prime}$ \\
\hline Coating layer & Nickel-Copper-Nickel Triple Layer \\
\hline $\mathrm{B}_{\mathrm{r}}$ & 13800 Gauss \\
\hline Pole Surfaces & $1^{\prime \prime} \times 1^{\prime \prime}$ surfaces \\
\hline Pull force & Over 54 lbs \\
\hline
\end{tabular}

Water proofing of the magnetic housing was done by welding with air gun and HDPE weld strips at around $500^{\circ} \mathrm{C}$ to $600^{\circ} \mathrm{C}$. To further enforce it, silicon resin coating was given. HDPE is light weight and has high strength to density ratio. Hence, it brings down the weight of the magnet array housing system. The advantage of using HDPE as material of construction over metals and PVC are as follows:

- HDPE is flexible compared to metal sheets or PVC sheets.

- It has low density and hence light in weight compared to metals. PVC is light but brittle.

- HDPE is non-corrosive whereas metals are corrosive and PVC has very poor chemical resistance.

- It can be welded easily when compared to metals and PVC materials.

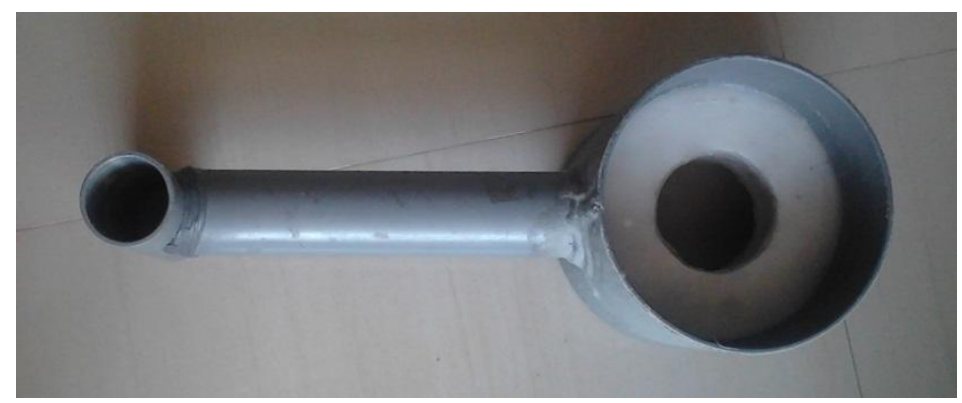

Figure 2: Magnet array housing 


\section{III.}

\section{CORE-COIL ASSEMBLy}

To increase the field strength for greater flux generation, an iron core was used. To prevent corrosion in case of presence of moisture, anti-corrosion silicon coating was provided to the core. Greater EMF is induced if the air gap between the core-coil assembly and magnet array housing is reduced to minimum. This was done by snuggly fitting the core-coil assembly surrounded by the magnet housing by using off-shelf outer covering made of poly vinyl chloride (PVC). Multilayer winding of copper wire was employed to make the core-coil diameter approximately equal to PVC outer covering diameter.Notches have to be made in the core to house the copper windings. The notches have to be first insulated with varnish or silicon coating and then by paper insulation.

The number of turns per winding is given as follows:

$N=\frac{\left(r_{o}-r_{i n}\right) w}{t^{2}}$

Where

$\mathrm{N}=$ number of turns per winding

$\mathrm{r}_{\mathrm{o}=}$ outer diameter of core

$\mathrm{r}_{\mathrm{in}}=$ inner diameter of core

$\mathrm{t}=$ thickness of wire

$\mathrm{w}=$ width of the notch

Two core-coil assemblies, assembly 1 and assembly 2 were manufactured. Assembly 1 was found to have a large air gap and this was overcome in assembly 2 by using a core of smaller diameter with spacers and by employing multilayer winding to decrease the distance between magnetic housing and core-coil assembly. With decreased air-gap, assembly 2 saw a phenomenal rise in output voltage. This increase in the output voltage can also be attributed to the increase in number of turns per winding. The dependence of induced voltage on the turns is given as follows:

$V(t)=N \frac{d \phi}{d t}$

Where

$\mathrm{N}=$ the number of turns

$\phi=$ the magnetic flux and

$\mathrm{V}(\mathrm{t})=$ the induced EMF.

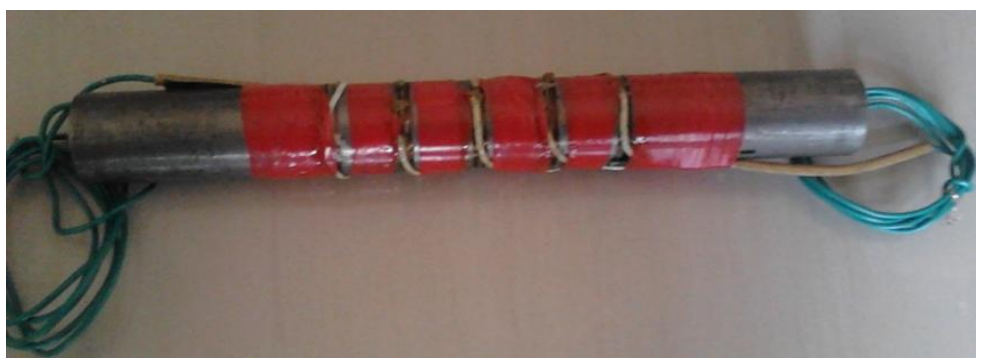

Figure 3: Core-coil assembly

Table 2: Coil Specifications

\begin{tabular}{|l|l|}
\hline Coil Specification & Specification Value \\
\hline Material & Copper \\
\hline Exterior finish & Enamel coated \\
\hline Type & Single conductor wire \\
\hline Gauge(AWG) & 28 \\
\hline Temperature range & Upto $+200^{\circ} \mathrm{C}$ \\
\hline Turns & $1000 / 6000($ assembly $1 / 2)$ \\
\hline
\end{tabular}


IV.

EXPERIMENT RESULTS

The two core-coil assemblies were tested with the neodymium magnet array housing and the flowing results were obtained.

Table 3: Experiment Results

\begin{tabular}{|l|l|l|}
\hline Parameter & Core-Coil Assembly 1 & Core-Coil Assembly 2 \\
\hline Number of windings & 5 & 5 \\
\hline Total number of turns & 1000 & 6000 \\
\hline Insulation & Varnish and paper insulation & Varnish and paper insulation \\
\hline Notches in metal core & Yes & No \\
\hline Spacers & No & Yes \\
\hline Core diameter & 1.5 inches & 1 inch \\
\hline Output voltage $(\mathrm{mV})$ & 80 & 800 \\
\hline
\end{tabular}

The above results were obtained by moving the magnetic array housing over the core-coil assembly at a rate of 20 times per minute.

\section{CONCLUSION}

This paper presents an optimized system which is capable of efficient conversion of mechanical energy into useful electrical energy, overcoming critical challenges.The output voltage of the linear generator was increased with increased number of turns and decrease in air-gap. Weight of the system and interference to the magnetic field was reduced with the use of HDPE.There is vast scope for future work. For larger loads, the linear generator's output power should range from few kilowatts to megawatts. The number of magnets and grade of magnets used has to be increased without large increase in weight as the magnet array housing moves up and down in water. Use of electromagnets has to be explored so that the magnetic field intensity can be controlled to the desired value. However, this poses a challenge as linear generators are suspended on river beds in large scale projects.

\section{Acknowledgements}

The authors would like to thank Mr. Gururaja Manur and Mr. Krishna Rao Badami for their technical assistance during the course of the project.

\section{REFERENCES}

[1] T. Sarpkaya, "Vortex-Induced Oscillations: A Selective Review,"Journal of Applied Mechanics, vol. 46, 1979, 241-258.

[2] R. A. Skop and O. M. Griffin, "A model for the vortex-excited resonant response of bluff cylinders," Journal of Sound andVibration, vol. 27, 1973, 225-233.

[3] C. H. K. Williamson and R. Govardhan, "Vortex-induced vibrations," Annual Review of Fluid Mechanics, vol. 36, 200, 413- 455

[4] R. D. Blevins, "Flow-induced vibrations," Journal of Sound and Vibration, vol. 149, 1990.

[5] Bernard T. Merritt, Robert F. Post, Gary R. Dreifuerst, Donald A. Bender;Halbach Array Motor/Generators-A Novel Generalized Electric Machine, Lawrence Livermore National Laboratory, UCRL-JC-119050, 1994. 